\title{
BMJ Open Acupuncture for neck pain caused by cervical spondylosis: a systematic review and meta-analysis protocol
}

\author{
Zhen Gao (D) , ${ }^{1}$ Gao-Feng Liu, ${ }^{2}$ Jing Zhang, ${ }^{3}$ Lai-Xi Ji ${ }^{4}$
}

To cite: Gao Z, Liu G-F, Zhang J, et al. Acupuncture for neck pain caused by cervical spondylosis: a systematic review and metaanalysis protocol. BMJ Open 2020;10:e038455. doi:10.1136/ bmjopen-2020-038455

- Prepublication history for this paper is available online. To view these files, please visit the journal online (http://dx.doi org/10.1136/bmjopen-2020038455).

Received 12 March 2020 Revised 14 0ctober 2020 Accepted 12 November 2020

Check for updates

(C) Author(s) (or their employer(s)) 2020. Re-use permitted under CC BY-NC. No commercial re-use. See rights and permissions. Published by BMJ.

${ }^{1}$ Acupuncture and Tuina School/ The 3rd Teaching Hospital, Chengdu University of Traditional Chinese Medicine, Chengdu,

China

${ }^{2}$ Graduate Faculty, Tianjin University of Traditional Chinese Medicine, Tianjin, China

${ }^{3}$ Department of acupuncture, Affiliated Hospital of Shanxi University of Traditional Chinese Medicine, Taiyuan, China

${ }^{4}$ The 3rd Teaching Hospital, Shanxi University of Traditional Chinese Medicine, Jinzhong, China

Correspondence to Lai-Xi Ji; tyjilaixi@126.com

\section{ABSTRACT}

Introduction Neck pain causes serious social and economic burden. Research on the use of acupuncture for managing cervical spondylosis has increased over time, with the quality of studies showing an improved trend. The present study seeks to use a systematic review approach to understand efficacy and safety of acupuncture for treatment of neck pain caused by cervical spondylosis. Methods and analysis We will search PubMed, Web of Science, Cochrane Library, Embase, China National Knowledge Infrastructure, Chinese BioMedical Literature, Wanfang database and VIP databases, from their inception to July 2020, to identify and retrieve all randomised controlled trials, describing the use of acupuncture for treatment of cervical spondylosis. Thereafter, two reviewers will independently select the studies, extract data and assess the risk of bias. Any disagreements, between them, will be resolved through a discussion with a third reviewer. Data synthesis and statistical analyses will be performed using the Revman V.5.3 software. Specifically, data will be synthesised by either fixedeffects (heterogeneity less than $50 \%$ ) or random-effects models, following a heterogeneity test, with outcome measures focusing on pain intensity, functional disability, psychological improvements and adverse events. In cases where no considerable heterogeneity is detected, a metaanalysis will be conducted.

Ethics and dissemination No ethical approval will be required for this study, since it does not infringe on anyone's interests. The findings will be published in a peer-reviewed journal or disseminated through conferences.

PROSPERO registration number CRD42020152379.

\section{INTRODUCTION}

Neck pain (NP) is a prevalent musculoskeletal disorder. The annual prevalence rate of NP exceeds $30 \%$, making it the fourth leading cause of disability. ${ }^{1} \mathrm{NP}$ is a multifactorial disease being associated with various dysfunctions, among them is cervical spondylosis which causes NP. ${ }^{2}$ The Chinese Association of Rehabilitation Medicine defines cervical spondylosis as a series of clinical syndromes resulting from degenerative changes of cervical intervertebral disc and secondary pathological changes involving surrounding tissue structures (nerve root, spinal cord,

\section{Strengths and limitations of the study}

The protocol was written in accordance with the Preferred Reporting Items for Systematic Reviews and Meta-Analyses Protocol guidelines.

- We will use a broad and comprehensive search strategy to search multiple electronic databases.

- To ensure that the included studies are free of personal bias, study selection, data extraction and bias risk assessment will be performed independently by two reviewers.

- The special nature of acupuncture implies that it will be difficult to perform a double-blind study.

- We anticipate possible heterogeneity among the studies to be included, owing to existence of multiple types of cervical spondylosis.

vertebral artery, sympathetic nerve). On the basis of clinical symptoms, signs and imaging tests (X-ray film, functional magnetic resonance), cervical spondylosis can be divided into nerve root type (ICD-10-CM G54.251), vertebral artery type (ICD-10-CM M47.025+) and other types. ${ }^{3}$ Previous studies showed that age, ${ }^{4}$ occupational factors, ${ }^{5}{ }^{6}$ ongoing postures and/or repetitive movements contribute to the development of spondylosis. ${ }^{7}$ In recent years, with the change in people's lifestyles, the age of onset of this condition has decreased. ${ }^{8}$ Cervical spondylosis has become an important healthcare and social problem.

Due to the complexity of its aetiology and high recurrence, the targets of treatment options for NP are poor. Evidence shows that studies on the analgesic efficacy of interventional therapy are mixed, especially surgery and cervical epidural injections. ${ }^{9-12}$ Some medicines (eg, muscle relaxants and nonsteroidal anti-inflammatory drugs) have been effective in alleviating acute pain, but its side effects cannot be ignored. ${ }^{13}$ It is therefore imperative to explore and develop complementary and alternative therapies to improve patient outcomes. So far, therapies such as lifestyle interventions, ${ }^{14}$ exercise, ${ }^{15} 16$ 
psychological therapies ${ }^{17}$ and ergonomical interventions have been proven to have a favourable risk ratio (RR) and availability ${ }^{18} 19$ Among them, acupuncture, a component of traditional Chinese medicine, has been extensively used in the treatment of pain for more than 3000 years. ${ }^{20}$ In practice, acupuncture involves a series of procedures, including inserting a filament needle into a specific acupuncture point, and then stimulating the acupuncture point by adjusting the needle.

Studies suggest that acupuncture confers analgesic effects through various mechanisms. In terms of the neurophysiological mechanisms, acupuncture activates the activating endogenous antinociceptive systems and decremental suppression systems to relieve pain. ${ }^{21}$ This is achieved via interaction and integration of the two different sensations of the acupuncture signal and the pain signal at different levels of the central nervous system. In terms of the neurochemical mechanisms, a series of studies have shown that interactions between different 'classical' neurotransmitters in the prefrontal cortex mediate the inhibition of pain perception. ${ }^{22}$ 5-hydroxytryptamine (serotonin) have been implicated in the modulation of central and peripheral pain. Similar to serotonin, norepinephrine (NE) is an important transmitter of the postganglia sympathetic nerve and many other central neurons. The upper and lower NE fibres project to the brain and spinal cord, respectively, and collectively they participate in the analgesic effects of acupuncture.$^{23}$ In addition, compelling evidence suggests that acupuncture can increase the release of opioid peptides in the brain, regulate the content of $\beta$-endorphin $(\beta-\mathrm{EP})$, and induce analgesia. ${ }^{24}{ }^{25}$ This indicates that acupuncture is an effective treatment option for NP.

However, the effect of acupuncture on NP seems controversial based on evidence-based perspective. Several systematic reviews (SRs) have been carried out to assess the clinical benefits of acupuncture therapy for NP, but findings from such studies have been inconclusive. A review conducted by Cagnie et al concluded that acupuncture was more effective than placebo intervention, but similar to other interventions. ${ }^{26}$ Even more, Smith et al suggested that it was no more effective than the placebo. ${ }^{27}$ Furthermore, inconsistent results have been reported across SRs with regard to differences between acupuncture and other interventions. ${ }^{28-30}$ Recently, it has been noticed with great interest that some new randomised controlled trials (RCTs) regarding acupuncture in cervical spondylosis have been published. ${ }^{31-34}$ Thus, we present this SR protocol designed to investigate the efficacy and safety of acupuncture for NP caused by cervical spondylosis.

\section{MATERIAL AND METHODS}

\section{Protocol and registration}

The protocol was developed according to the Preferred Reporting Items for Systematic Reviews and Meta-Analyses Protocols (PRISMA-P) guidelines. The review will be conducted in accordance with the PRISMA guidelines. ${ }^{35}$
The study is expected to begin searching in July 2020 and end in March 2021.

\section{Criteria for including studies for the review \\ Types of studies}

We will include all RCTs that evaluated acupuncture therapy for NP caused by cervical spondylosis. To avoid risk of bias, crossover trials, cluster-RCTs, quasi-RCTs or trials without control group will be excluded. Only studies written in English and Chinese will be included.

\section{Types of participants}

Participants diagnosed with cervical spondylosis will be included. Diagnostic criteria will include the International Statistical Classification of Diseases and HealthRelated Problems, ${ }^{36}$ Treatment and Rehabilitation of Cervical Spondylosis. Participants with cervical spondylosis without NP will be excluded. No restrictions will be applied in terms of age, gender and ethnicity.

\section{Types of interventions}

Acupuncture therapies will include body acupuncture, manual acupuncture, warm acupuncture, ear acupuncture, plum blossom needling, fire needling and electroacupuncture. However, studies investigating transcutaneous electrical nerve stimulation, acupressure and laser stimulation as acupuncture interventions will be excluded. In addition, studies comparing between different types of acupuncture therapies will be excluded.

\section{Types of comparator(s)/control}

Interventions to be compared will include sham acupuncture (interventions mimicking 'verum' acupuncture, but do not pierce the skin), waiting list control, herbal medicine, moxibustion, wet cupping, western medicine, usual care and psychological intervention. In addition, any control group who underwent skin penetration by needles will be excluded. Furthermore, we will include studies that compared acupuncture combined with any other type of non-acupuncture versus non-acupuncture intervention.

\section{Type of outcome measures}

\section{Primary outcomes}

Pain intensity will be measured using scales such as the Visual Analogue Scale, Northwick Park Questionnaire and Numeric Rating Scale.

\section{Secondary outcomes}

1. Functional disability, measured by the Neck Disability Index and Oswestry Disability Index.

2. Psychological improvements, measured by scales such as the Hamilton Anxiety Rating Scale and Self-rating Anxiety Scale.

3. Adverse events.

\section{Patient and public involvement}

The study was a non-clinical trial that did not seek the participation of patients or the public. 


\begin{tabular}{|c|c|c|c|}
\hline \multicolumn{2}{|c|}{ Table 1 Search strategy in PubMed } & \multicolumn{2}{|c|}{ Table 1 Continued } \\
\hline$\# 1$ & pain. Mesh & \multirow{2}{*}{ \#49 } & \multirow{2}{*}{$\begin{array}{l}\# 37 \text { or \#38 or \#39 or \#40 or \#41 or \#42 or \#43 or \#44 or \#45 or } \\
\# 46 \text { or \#47 or \#48 }\end{array}$} \\
\hline \#2 & pain. ti, ab & & \\
\hline \#3 & ache. ti, ab & \#50 & randomize controlled trial. Pt \\
\hline \#4 & sore. ti, ab & \#51 & controlled clinical trial. Pt \\
\hline \#5 & stiff. ti, ab & \#52 & randomize. ti, ab \\
\hline \#6 & discomfort. ti, ab & \#53 & Placebo. ti, ab \\
\hline \#7 & $\# 1$ or \#2 or \#3 or \#4 or \#5 or \#6 & \#54 & randomly. ti, ab \\
\hline \#8 & neck pain. Mesh & \#55 & trial. Ti \\
\hline \#9 & neck pain. ti, ab & \#56 & $\# 50$ or \#51 or \#52 or \#53 or \#54 or\#55 \\
\hline \#10 & neck disorder. ti, ab & \#57 & humans. Mh \\
\hline \#11 & neck muscles. ti, ab & \#58 & $\# 56$ and \#57 \\
\hline \#12 & neckache. ti, ab & \#59 & \#36 and \#49 and \#58 \\
\hline
\end{tabular}

\section{Search methods for identification of studies}

The following electronic databases: PubMed, Web of Science, Cochrane Library, Embase, China National Knowledge Infrastructure, Chinese BioMedical Literature, Wanfang database and VIP Database will be searched from inception to July 2020. In addition, we will scrutinise the list of references, relevant conference literature and trial registry database (WHO International Clinical Trials Registry Platform and Clinical Trials.gov) to identify additional studies.

The search strategy for PubMed is shown in table 1. The following search terms will be used singly or as combinations (MeSH terms and free words): pain, acupuncture, acupuncture therapy and randomised controlled trial. The search terms will be translated into Chinese for study identification in Chinese databases.

\section{Data collection and analysis}

Selection of studies

Two reviewers will be required to screen the retrieved studies independently. Briefly, they will exclude duplicate studies and those not matching the inclusion criteria by reading title and abstracts. They will read the full text of each study to select those meeting the inclusion criteria. Any disagreements will be resolved through discussion with a third reviewer. The entire study selection process is shown in the flow diagram (figure 1).

\section{Data extraction}

Data extraction from the included studies will be done independently by two reviewers following a data acquisition list. The list will include the basic information (author, title, journal, year of publication and country of publication), study design (study size, randomisation, allocation concealment, blinding methods, type of interventions and treatment duration), outcome measures and conflicts of interest. If necessary, a third reviewer will double-check the data to ensure consistency.

If data are missing or incomplete in any study, we will contact with the authors to obtain such data. In case it is impossible to obtain the data, to ensure the statistical power and avoid bias, the last observation carried forward 

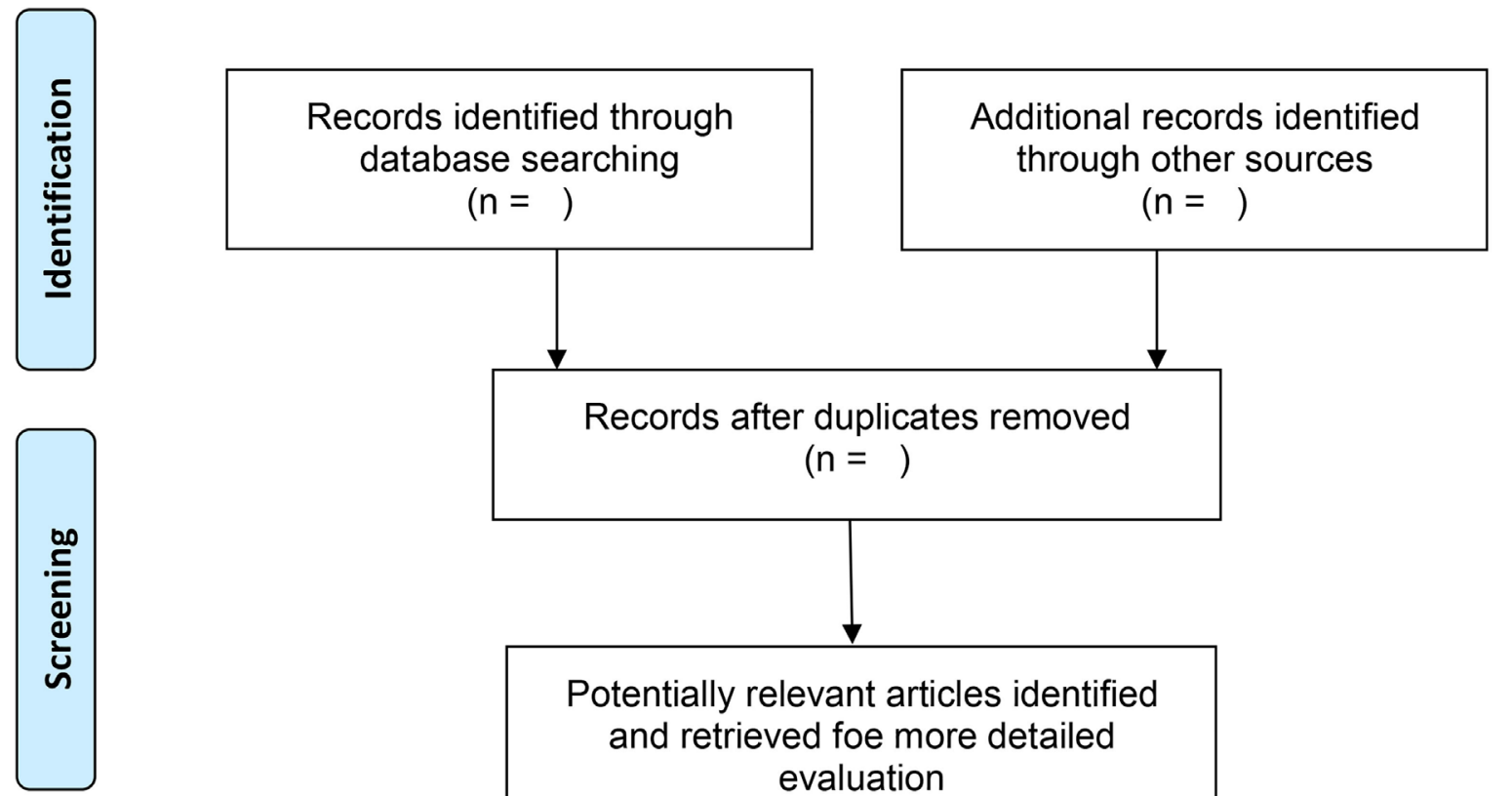

Records after duplicates removed

$$
(n=)
$$

Additional records identified through other sources

$$
(n=)
$$
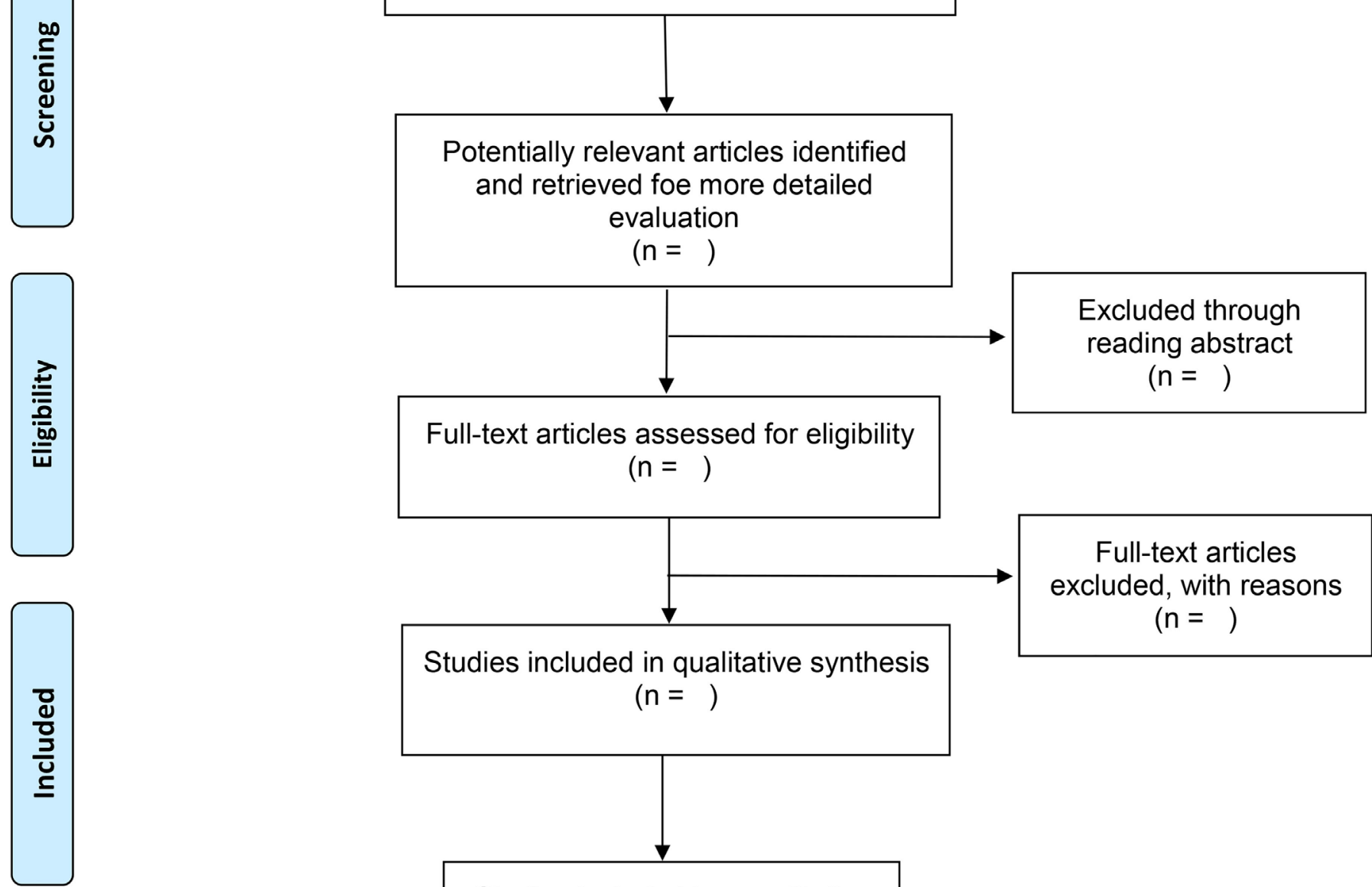

\section{Full-text articles assessed for eligibility} $(n=)$

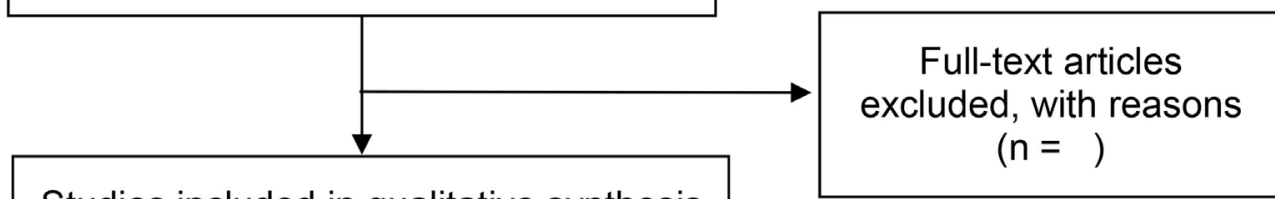

Studies included in qualitative synthesis $(n=)$

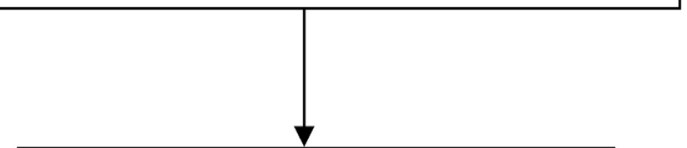

Studies included in quantitative synthesis (meta-analysis) $(n=)$

Figure 1 Flow diagram of the study design.

imputation method will be used to assume a missing value and then an intent-to-treat analysis and sensitivity analysis will be performed to assess whether that the results are consistent.

\section{Assessment of risk of bias}

The risk of bias in the included studies will be determined by two reviewers independently using the Cochrane Collaboration tool. ${ }^{37}$ The Cochrane Collaboration's tool covers six aspects, sequence generation, allocation concealment, blinding, incomplete outcome data, selective reporting and other bias. The risk will be divided into three levels (low risk, high risk and unclear) in accordance with the item in the checklist. If any disagreements, the risk assignment will be settled through arbitration of a third reviewer.

\section{Measures of treatment effect}

For continuous outcome data, the standardised mean difference or weighted mean difference with $95 \%$ CIs will be used. For dichotomous data, the RR with $95 \%$ CIs will be used for analysis. 


\section{Assessment of heterogeneity}

We will evaluate heterogeneity using the standard $\chi^{2}$ test $(\alpha=0.1)$ and $\mathrm{I}^{2}$ test, if the $\mathrm{p} \geq 0.1$, and if $\mathrm{I}^{2} \leq 50 \%$, the fixedeffects model will be used. Random-effects models will be used if the $\mathrm{p}<0.1$ or the $\mathrm{I}^{2}>50 \%$. A subgroup analysis will be performed to explore the possible causes. If the heterogeneity is greater than $75 \%$, a meta-analysis will not be performed. We will provide a narrative, qualitative summary.

\section{Assessment of reporting biases}

We will use funnel plots to demonstrate potential reporting biases in cases involving more than 10 studies.

\section{Subgroup analysis}

If data are available, subgroup analysis will be performed to assess the heterogeneity according to the types of interventions, types of cervical spondylosis, acupuncture frequency and different outcomes.

\section{Sensitivity analysis}

If possible, sensitivity analysis will be used to evaluate how uncertain assumptions of data and usage affect the robustness of the combined results. We will exclude lowquality studies, reanalyse the included studies, and assess whether there are significant differences between the combined effects.

\section{Grading the quality of evidence}

The quality of the evidence will be used the Grading of Recommendations Assessment, Development and Evaluation (GRADE) ${ }^{37}$ to assess the quality of evidence for each outcome. The following criteria will be applied: design of the study, soundness of implementation, imprecision and indirectness of evidence, consistency or homogeneity of the results and other biases. The level of evidence will be classified into four: 'high', 'moderate', 'low' or 'very low' according to the GRADE rating standards.

\section{Ethics and dissemination}

This review will not require ethical approval as it does not infringe on anyone's interests. The results will be published in a peer-reviewed journal or disseminated through conferences.

\section{DISCUSSION}

Cervical spondylosis has a high prevalence, and greatly affects quality of life in patients with such condition. Acupuncture might be effective for the treatement of cervcial spondylosis, and findings of this update to former SRs are intended to provide state-of-the-art overview.

Contributors ZG is responsible for the conception and design of research, the formulation of retrieval strategies, data extraction and analysis and article writing; G-FL participates in the formulation of retrieval strategies, data acquisition and article revision; JZ participates in data extraction, analysis and interpretation, article revision; L-XJ participated in the formulation of search strategies and critical revision of articles. All authors contributed to the research and agreed to be responsible for all aspects of the work.
Funding This study was supported by the National Natural Science Foundation of China (no. 81673887), the National Science and Technology Support Program of the ministry of science and technology of China (2013BAI05B00).

\section{Competing interests None declared.}

Patient and public involvement Patients and/or the public were not involved in the design, or conduct, or reporting or dissemination plans of this research.

Patient consent for publication Not required.

Provenance and peer review Not commissioned; externally peer reviewed.

Open access This is an open access article distributed in accordance with the Creative Commons Attribution Non Commercial (CC BY-NC 4.0) license, which permits others to distribute, remix, adapt, build upon this work non-commercially, and license their derivative works on different terms, provided the original work is properly cited, appropriate credit is given, any changes made indicated, and the use is non-commercial. See: http://creativecommons.org/licenses/by-nc/4.0/.

ORCID iD

Zhen Gao http://orcid.org/0000-0001-8410-8630

\section{REFERENCES}

1 Cohen SP. Epidemiology, diagnosis, and treatment of neck pain. Mayo Clin Proc 2015;90:284-99.

2 Vogt MT, Cawthon PM, Kang JD, et al. Prevalence of symptoms of cervical and lumbar stenosis among participants in the osteoporotic fractures in men study. Spine 2006;31:1445-51.

3 Ahmed SB, Qamar A, Imram M, et al. Comparison of neck length, relative neck length and height with incidence of cervical spondylosis. Pak J Med Sci 2020;36:219-23.

4 Wang $C$, Tian F, Zhou Y, et al. The incidence of cervical spondylosis decreases with aging in the elderly, and increases with aging in the young and adult population: a hospital-based clinical analysis. Clin Interv Aging 2016;11:47-53.

$5 \mathrm{Kwon} \mathrm{Y}, \mathrm{Kim} \mathrm{J}-\mathrm{W}, \mathrm{Heo} \mathrm{J}-\mathrm{H}$, et al. The effect of sitting posture on the loads at cervico-thoracic and lumbosacral joints. Technol Health Care 2018;26:409-18.

6 Singla D, Veqar Z. Association between forward head, Rounded shoulders, and increased thoracic kyphosis: a review of the literature. J Chiropr Med 2017;16:220-9.

7 Lv Y, Tian W, Chen D, et al. The prevalence and associated factors of symptomatic cervical spondylosis in Chinese adults: a community-based cross-sectional study. BMC Musculoskelet Disord 2018;19:325.

8 Smith L, Louw Q, Crous L, et al. Prevalence of neck pain and headaches: impact of computer use and other associative factors. Cephalalgia 2009;29:250-7.

9 Boswell MV, Hansen HC, Trescot AM, et al. Epidural steroids in the management of chronic spinal pain and radiculopathy. Pain Physician 2003;6:319-34.

10 Peng B, DePalma MJ. Cervical disc degeneration and neck pain. $J$ Pain Res 2018;11:2853-7.

11 Mesregah MK, Feng W, Huang W-H, et al. Clinical effectiveness of Interlaminar epidural injections of local anesthetic with or without steroids for managing chronic neck pain: a systematic review and meta-analysis. Pain Physician 2020;23:335-48.

12 Manchikanti L, Nampiaparampil DE, Candido KD, et al. Do cervical epidural injections provide long-term relief in neck and upper extremity pain? A systematic review. Pain Physician 2015;18:39-60.

13 Cohen SP, Hooten WM. Advances in the diagnosis and management of neck pain. BMJ 2017;358:j3221.

14 Gordon SJ, Grimmer KA, Buttner P. Pillow preferences of people with neck pain and known spinal degeneration: a pilot randomized controlled trial. Eur J Phys Rehabil Med 2019;55:783-91.

15 Blanpied PR, Gross AR, Elliott JM, et al. Neck pain: revision 2017. J Orthop Sports Phys Ther 2017;47:A1-83.

16 Pereira M, Comans T, Sjøgaard G, et al. The impact of workplace Ergonomics and neck-specific exercise versus Ergonomics and health promotion interventions on office worker productivity: a cluster-randomized trial. Scand J Work Environ Health 2019;45:42-52.

17 Williams ACdeC, Eccleston C, Morley S. Psychological therapies for the management of chronic pain (excluding headache) in adults. Cochrane Database Syst Rev 2012;11:Cd007407.

18 Tsang SMH, So BCL, Lau RWL, et al. Effects of combining ergonomic interventions and motor control exercises on muscle 
activity and kinematics in people with work-related neck-shoulder pain. Eur J Appl Physiol 2018;118:751-65.

19 de Campos TF, Maher CG, Steffens D, et al. Exercise programs may be effective in preventing a new episode of neck pain: a systematic review and meta-analysis. J Physiother 2018;64:159-65.

20 Robinson N, Lorenc A, Ding W, et al. Exploring practice characteristics and research priorities of practitioners of traditional acupuncture in China and the EU-A survey. J Ethnopharmacol 2012;140:604-13.

21 Irnich D, Beyer A. [Neurobiological mechanisms of acupuncture analgesia]. Schmerz 2002;16:93-102.

22 Ong W-Y, Stohler CS, Herr DR. Role of the prefrontal cortex in pain processing. Mol Neurobiol 2019;56:1137-66.

23 Zhang Y, Zhang RX, Zhang M, et al. Electroacupuncture inhibition of hyperalgesia in an inflammatory pain rat model: involvement of distinct spinal serotonin and norepinephrine receptor subtypes. $\mathrm{Br} \mathrm{J}$ Anaesth 2012;109:245-52.

24 Gao Y-H, Wang J-Y, Tan L-H, et al. [High Mobility Group Box 1/ CD 24 Receptor/ $\beta$-EP Signaling in "Zusanli" (ST 36) Region Contributes to Electroacupuncture Analgesia in Rats with Neuropathic Pain]. Zhen Ci Yan Jiu 2018;43:537-42.

25 Han J-S. Acupuncture and endorphins. Neurosci Lett 2004;361:258-61.

26 Cagnie B, Castelein B, Pollie F, et al. Evidence for the use of ischemic compression and dry Needling in the management of trigger points of the upper trapezius in patients with neck pain: a systematic review. Am J Phys Med Rehabil 2015;94:573-83.

27 Smith LA, Oldman AD, McQuay HJ, et al. Teasing apart quality and validity in systematic reviews: an example from acupuncture trials in chronic neck and back pain. Pain 2000;86:119-32.

28 Trinh K, Graham N, Irnich D, et al. Acupuncture for neck disorders. The Cochrane database of systematic reviews 2016:5:Cd004870.
29 Yuan Q-ling, Guo T-mao, Liu L, et al. Traditional Chinese medicine for neck pain and low back pain: a systematic review and meta-analysis. PLOS One 2015;10:e0117146.

30 Fu L-M, Li J-T, Wu W-S, et al. Randomized controlled trials of acupuncture for neck pain: systematic review and meta-analysis. $J$ Altern Complement Med 2009;15:133-45.

31 Ho LF, Lin ZX, Leung AWN, et al. Efficacy of abdominal acupuncture for neck pain: a randomized controlled trial. PLoS One 2017;12:e0181360.

32 Xie J-mou, Chen Z-qiang, Guo W, et al. [Efficacy observation of cervical spondylosis treated with acupuncture at three lines of cervical Jiaji (EX-B 2)]. Zhongguo Zhen Jiu 2014;34:863-6.

33 Pan S-L, Zheng S-L, Zhou X-H, et al. [Acupuncture combined with Jingtong granule for nerve-root type cervical spondylosis and its effects on IL-6、TNF- $\alpha$, IL-1 $\beta$ and hemorheological indexes] Zhongguo Zhen Jiu 2019;39:1274-8.

34 Meng X-W, Wang Y, Piao S-A, et al. Wet cupping therapy improves local blood perfusion and analgesic effects in patients with nerveroot type cervical spondylosis. Chin J Integr Med 2018;24:830-4.

35 Liberati A, Altman DG, Tetzlaff J, et al. The PRISMA statement for reporting systematic reviews and meta-analyses of studies that evaluate health care interventions: explanation and elaboration. PLOS Med 2009;6:e1000100.

36 Organization WH. International statistical classification of diseases and related health problems: 10th revision (ICD-10), 2020. Available: https://apps. who.int/iris/handle/10665/43110?locale=fr

37 Guyatt GH, Oxman AD, Vist GE, et al. Grade: an emerging consensus on rating quality of evidence and strength of recommendations. $B M J$ 2008;336:924-6. 